\title{
Electromagnetic Biomedical Imaging in Banach Spaces: A Numerical Case Study
}

\author{
Alessandro Fedeli, Matteo Pastorino, and Andrea Randazzo \\ Department of Electrical, Electronic, Telecommunications Engineering and Naval Architecture (DITEN), \\ University of Genoa, Genoa, Italy.
}

\begin{abstract}
This paper reports the results of the application of a microwave imaging method developed in Banach spaces to a model of human head in presence of a hemorrhagic brain stroke. The approach is based on the integral equations of the inverse scattering problem. A Gauss-Newton scheme is adopted as a solving procedure. Being developed in Banach spaces, the method turns out to be quite efficient in reducing the over-smoothing effects usually associated to Hilbert-space reconstructions. Numerical simulations are reported involving a realistic model of human head.
\end{abstract}

\section{Introduction}

Microwave imaging techniques have been proposed for long time as potential diagnostic tools in biomedical applications [1]-[4]. One of the unresolved questions related to these techniques concerns the availability of effective inversion methods. Since the inverse scattering problem related to microwave imaging is, as it is well known, nonlinear and strongly ill-posed, proper regularization methods must be applied [5]. In the scientific literature, a plethora of inversion procedures have been proposed and assessed. In order to provide "quantitative" reconstructions of the dielectric properties of the target under test, the integral equations of the inverse scattering problem must be solved without approximations (apart from the numerical ones).

Among the various proposed methods, Gauss-Newton techniques seem to be quite efficient ones [6], [7]. However, they are usually developed in the framework of Hilbert spaces. Therefore, they suffer from over-smoothing effects, which can limit the accuracy of reconstructions, especially in biomedical applications. The present authors proposed in [8] the application to microwave imaging of a Gauss-Newton method developed in Banach spaces. The approach has been tested in several conditions [9], [10] and it has been found to provide accurate reconstructions of the considered scatterers.

In the present paper, the above approach, which is based on the integral equations of the inverse scattering problem, is applied to the detection of a hemorrhagic brain stroke in a numerical model of the human head. In particular, we consider a multi-illumination setup, in which a twodimensional slice of the Zubal model [11] is illuminated by a line current source and the scattered electric field is collected around the cross section of the target.
The paper is organized as follows. In Section 2, a brief outline of the considered approach is reported, whereas in Section 3 an example of the obtained results is provided.

\section{Outline of the proposed approach}

Let us consider the cross section $S$ of a cylindrical target illuminated under transverse magnetic conditions (a line current source parallel to the cylinder axis is used). Assuming to be able to measure the axial component of the electric field vector around the scatterer's cross section, $\Psi^{s}$, it is possible to relate these values with the dielectric properties of the scatterer under test. In particular, in case of time-harmonic fields, a functional equation can be written, i.e.,

$$
\Psi^{s}=\Xi\left(\tau, \Psi^{s}+\Psi^{i}\right)
$$

in which $\Psi^{i}$ denotes the axial component of the incident field vector inside the target and $\tau$ is the so-called object function, which directly depends on the excess of complex dielectric permittivity represented by the object with respect to the reference propagation medium.

In equation (1), the operator $\Xi(\cdot)$ is nonlinear and ill-posed, being representative of a Fredholm integral equation of the first kind having as a kernel the Green's function of the considered reference structure [12]. In order to invert equation (1) and retrieving the object function starting from measurements of the scattered electric field, some additional information must be used. In most cases, such information is included in an additional constraint in the model, i.e., the assumption that the object function must produce, inside the target, a scattered electric field consistent with the known incident field [1].

The nonlinear functional equation is solved by using a Gauss-Newton approach in Banach spaces [8]. In particular, this method is constituted by two nested loops. In the outer loop, Newton linearizations are performed, whereas in the inner loop the resulting ill-posed linear equation is regularized by using a conjugate gradient method. Since the whole approach is formulated in Banach spaces, it results to suffer less from over-smoothing effects in retrieving the dielectric profile of the target cross section.

\section{Numerical results}

In the case study that is analyzed throughout this paper, the above-mentioned approach has been applied to a numerical model of human head. In particular, the Zubal phantom has been considered [11]. In this section, we report some 
examples of the achieved results, obtained under a twodimensional approximation. With reference to the slice no. 40 of the Zubal head model, we simulated the presence of a hemorrhagic stroke by including inside the model an elliptical clot of blood located at $\mathbf{r}_{s}=(2.5,0) \mathrm{cm}$, with $x$ directed axis of length $s_{x}$, and $y$-directed axis $s_{y}=2 s_{x}$. The head is immersed in a lossless matching medium with relative dielectric permittivity $\epsilon_{r}^{m}=20$. All the involved biological tissues have been characterized with their fourth-order Cole-Cole parametric models [13]. A multistatic and multiview measurement system is placed around the simulated head, with $V=36$ measurement positions equally distributed along a circumference of diameter $d=22 \mathrm{~cm}$, centered at the origin. Sequentially, a line-source antenna occupies one of these positions and illuminates the scene, whereas all the remaining $M=V-$ $1=35$ locations are utilized for collecting measurements of the scattered electric field $\Psi^{s}$. The working frequency is $800 \mathrm{MHz}$. The total electric field values, computed by means of a numerical code implementing a method of moments formulation, are corrupted by an additive white Gaussian noise with zero mean value and a standard deviation that corresponds to a signal-to-noise ratio equal to $45 \mathrm{~dB}$. The investigation domain, which is an ellipse centered at the origin with minor axis $l_{x}=8 \mathrm{~cm}$ and major axis $l_{y}=10 \mathrm{~cm}$, has been discretized into 5199 square cells of side $0.22 \mathrm{~cm}$ for solving the forward electromagnetic problem, and 1300 cells of side $0.44 \mathrm{~cm}$ for solving the inverse one. As regards the inversion method parameters, 20 and 50 maximum steps in the outer and in the inner loops have been fixed, respectively. Moreover, iterations are stopped if the relative difference between the data residuals at two subsequent iterations decreases under a threshold of $0.5 \%$ for the outer loop, and $1 \%$ for the inner one.

Several numerical simulations with different dimensions of the brain stroke $s_{x}$ and values of the Banach space norm parameter $p$ [8] have been executed. The imaginary part of the reconstructed difference between the actual object function $\tau$ and the considered reference configuration (i.e., the healthy head), denoted as $\Delta \tau$, has been reported in Figure 1 and Figure 2 for two different brain stroke sizes. These reconstructions have been obtained with the Banach space norm parameter $p$ equal to 1.5 . As can be seen, in both cases the hemorrhagic brain stroke included in the head model is correctly detected and located. Figure 3 reports the average relative reconstruction error inside the region occupied by the brain stroke versus the Banach space norm parameter $p$, for different dimensions of the ellipse that has been used to model the stroke. Clearly, without a strong dependence on the stroke size, values of $p$ between 1.5 and 1.6 allow to obtain the best dielectric reconstructions of the stroke region. It is worth noting that the conventional Hilbert-space reconstructions are provided as a special case for $p=2$. The error on the stroke inclusion reconstruction decreases if the stroke size grows. The corresponding average relative reconstruction errors related to the background (i.e., the part of the investigation domain that does not belong to the brain stroke region) are presented in Figure 4. It can be noticed that the Banach space approach, especially with low values of $p$, greatly improves the reconstruction of the background region, which turns out to be more clean and free from artifacts and ringing effects. This trend is independent from the size of the considered hemorrhagic brain stroke. Furthermore, in this case, the background error rises with an increasing size of the brain stroke.

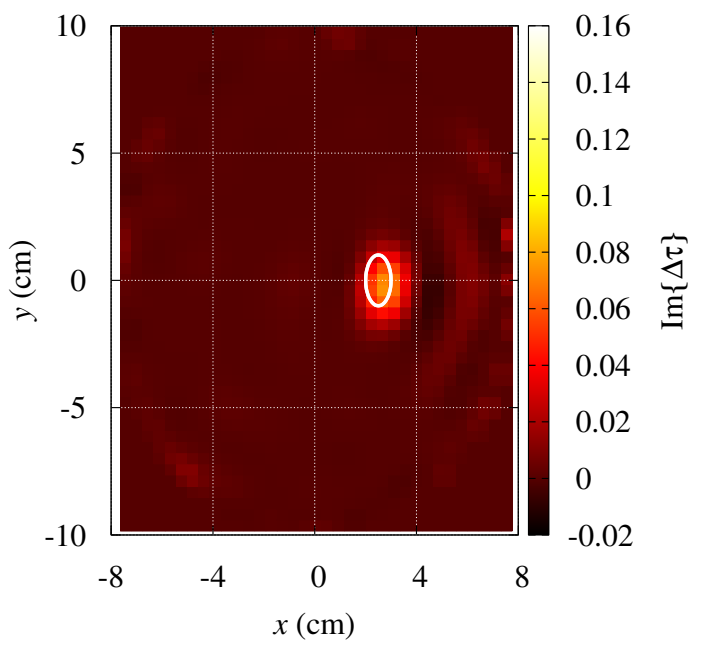

Figure 1. Reconstruction of a head model in presence of a hemorrhagic brain stroke with minor and major axis lengths $s_{x}=1 \mathrm{~cm}$ and $s_{x}=2 \mathrm{~cm}$, respectively. Distribution of the imaginary part of the differential object function $\Delta \tau$.

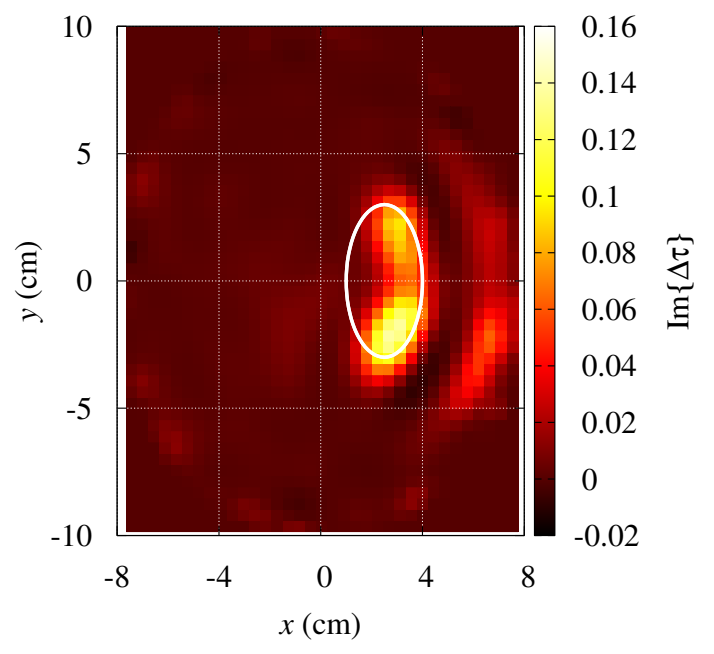

Figure 2. Reconstruction of a head model in presence of a hemorrhagic brain stroke with minor and major axis lengths $s_{x}=3 \mathrm{~cm}$ and $s_{x}=6 \mathrm{~cm}$, respectively. Distribution of the imaginary part of the differential object function $\Delta \tau$. 


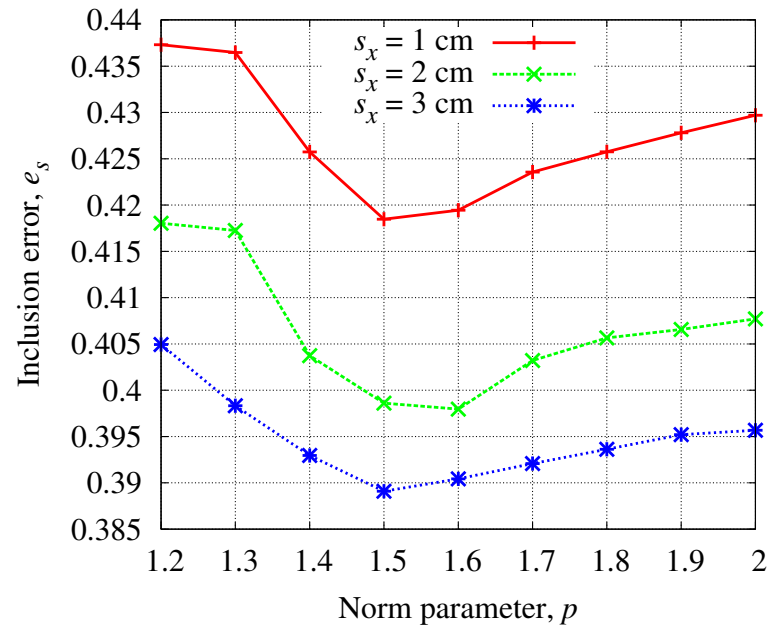

Figure 3. Relative reconstruction error on the region including the brain stroke versus different values of the Banach space norm parameter $p$, for some stroke dimensions.

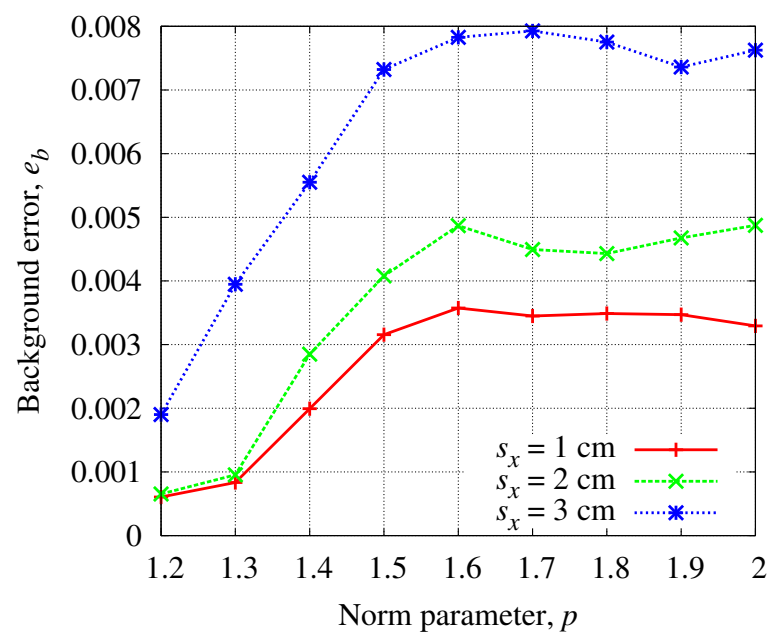

Figure 4. Relative reconstruction error on the background region versus different values of the Banach space norm parameter $p$, for some stroke dimensions.

\section{Acknowledgements}

The present work is partially supported by the "Compagnia di San Paolo", Italy.

\section{References}

1. M. Pastorino, Microwave imaging. Hoboken, N.J.: John Wiley \& Sons, 2010.

2. R. O. Mays, N. Behdad, and S. C. Hagness, "A TSVD Analysis of the Impact of Polarization on Microwave Breast Imaging Using an Enclosed Array of Miniaturized Patch Antennas," IEEE Antennas Wirel. Propag. Lett., 14, 2015, pp. 418-421.
3. K. Bialkowski, D. Ireland, and A. Abbosh, "Microwave imaging for brain stroke detection using Born iterative method," IET Microw. Antennas Propag., 7, 11, Aug. 2013, pp. 909-915.

4. M. Persson, A. Fhager, H. D. Trefna, Y. Yu, T. McKelvey, G. Pegenius, J.-E. Karlsson, and M. Elam, "Microwave-Based Stroke Diagnosis Making Global Prehospital Thrombolytic Treatment Possible," IEEE Trans. Biomed. Eng., 61, 11, Nov. 2014, pp. 2806-2817.

5. D. Colton and R. Kress, Inverse acoustic and electromagnetic scattering theory, 93. New York, NY: Springer New York, 2013.

6. T. Rubek, P. M. Meaney, P. Meincke, and K. D. Paulsen, "Nonlinear microwave imaging for breast-cancer screening using Gauss-Newton's method and the CGLS inversion algorithm," IEEE Trans. Antennas Propag., 55, 8, Aug. 2007, pp. 2320-2331.

7. M. Ostadrahimi, P. Mojabi, A. Zakaria, J. LoVetri, and L. Shafai, "Enhancement of Gauss-Newton inversion method for biological tissue imaging," IEEE Trans. Microw. Theory Tech., 61, 9, Sep. 2013, pp. 3424-3434.

8. C. Estatico, M. Pastorino, and A. Randazzo, "A novel microwave imaging approach based on regularization in Lp Banach spaces," IEEE Trans. Antennas Propag., 60, 7, Jul. 2012, pp. 3373-3381.

9. C. Estatico, A. Fedeli, M. Pastorino, and A. Randazzo, "Buried object detection by means of a Lp Banach-space inversion procedure," Radio Sci., 50, 1, Jan. 2015, pp. 4151 .

10. C. Estatico, A. Fedeli, M. Pastorino, and A. Randazzo, "A multifrequency inexact-Newton method in Lp Banach spaces for buried objects detection," IEEE Trans. Antennas Propag., 63, 9, Sep. 2015, pp. 4198-4204.

11. I. G. Zubal, "Computerized three-dimensional segmented human anatomy," Med. Phys., 21, 2, 1999, p. 299.

12. S. Caorsi, A. Massa, M. Pastorino, A. Randazzo, and A. Rosani, "A reconstruction procedure for microwave nondestructive evaluation based on a numerically computed Green's function," IEEE Trans. Instrum. Meas., 53, 4, Aug. 2004, pp. 987-992.

13. S. Gabriel, R. W. Lau, and C. Gabriel, "The dielectric properties of biological tissues: III. Parametric models for the dielectric spectrum of tissues," Phys. Med. Biol., 41, 11, Nov. 1996, pp. 2271-2293. 